\title{
INTERVAL-TYPE THEOREMS CONCERNING QUASI-ARITHMETIC MEANS
}

\author{
PAWEŁ PASTECZKA
}

Abstract. Family of quasi-arithmetic means has a natural, partial order (point-wise order) $A^{[f]} \leqslant$ $A^{[g]}$ if and only if $A^{[f]}(v) \leqslant A^{[g]}(v)$ for all admissible vectors $v(f, g$ and, later, $h$ are continuous, monotone and defined on a common interval).

Therefore one can introduce the notion of interval-type sets (sets $\mathscr{I}$ such that whenever $A^{[f]} \leqslant A^{[h]} \leqslant A^{[g]}$ for some $A^{[f]}, A^{[g]} \in \mathscr{I}$ then $A^{[h]} \in \mathscr{I}$ too).

Our aim is to give examples of interval-type sets involving vary smoothness assumptions of generating functions.

Mathematics subject classification (2010): Primary 26E60, Secondary 26D15.

Keywords and phrases: Quasi-arithmetic means, sandwich theorems, Arrow-Pratt index, comparability, smoothness assumptions.

\section{REFERENCES}

[1] B. DE FInetTI, Sul concetto di media, Giornale dell' Instituto, Italiano degli Attuarii 2, 1 (1931), 369-396.

[2] K. Knopp, Über Reihen mit positiven Gliedern, J. London Math. Soc. 3, 1 (1928), 205-211.

[3] A. N. Kolmogorov, Sur la notion de la moyenne, Rend. Accad. dei Lincei 12, 6 (1930), 388-391.

[4] J. G. Mikusińs Ki, Sur les moyennes de la forme $\psi^{-1}\left[\sum q \psi(x)\right]$, Studia Mathematica 10, 1 (1948), 90-96.

[5] M. Nagumo, Über eine Klasse der Mittelwerte, Jap. Jour. of Math. 7, 1 (1930), 71-79.

[6] P. PAsteCZKA, A new estimate of the difference among quasi-arithmetic means, Math. Inequal. Appl. 18, 4 (2015), 1321-1327.

[7] P. PasteczKa, Interval-type theorems concerning means, Ann. Univ. Paedagog. Crac. Stud. Math. 17, 1 (2018), 37-43.

[8] Zs. PÁLES, On the convergence of means, J. Math. Anal. Appl. 156, 1 (1991), 52-60. 